\title{
A scalarization-based method for multiple part-type scheduling of two-machine robotic systems with non- destructive testing technologies
}

\begin{abstract}
Mehdi. Foumani ${ }^{1}$, Reza. Tavakkoli-Moghaddam ${ }^{2, *}$
This paper analyzes the performance of a robotic system with two machines in which machines are configured in a circular layout and produce non-identical parts repetitively. The non-destructive testing (NDT) is performed by a stationary robotic arm located in the center of the circle, or a cluster tool. The robotic arm integrates multiple tasks, mainly the NDT of the part and its transition between a pair of machines. The robotic arm cannot complete the transition if it identifies a fault in the part. The main feature of the NDT technology is that its required time is changed by altering the testing cost. This generates a trade-off between cost and cycle time. Initially, the problem of robotic arm scheduling and part sequencing is jointly solved to supports the decision making for reliability improvement of small-scale robotic systems with NDT technologies. We show how the case of non-identical parts can be converted into a travelling salesman problem (TSP). Then, we provide a generalization of the framework based on three characteristics: pickup criterion, layout and travel time metric. The results are extended for the interval and no-wait pickup criteria, and then some notes are provided for travel time saving of different layout and travel time metric. It is shown whether circular systems are equivalent to linear systems, or they dominate linear cases in general terms.
\end{abstract}

Keywords: Performance analysis, Robotic systems, Non-destructive testing, Scalarization

Manuscript was received on 20/05/2018, revised on 14/11/2018 and accepted for publication on 23/04/2019.

\section{Introduction}

A robotic serial manufacturing system typically includes a material handler robotic arm and a set of different machines, such that the processing route of all parts on these machines is similar and it includes a chain of series operations. Note that parts are not often identical although their processing route on these machines is similar. This is because multiple part types belong to the same part family [1]. Therefore, the case of multiple part types is closer to the reality. Also, to make this robotic system better represent reality, the testing process should be used in the system including the robotic arm and machines. This is because poor quality final products can even destroy the reputation of the manufacturer through out-situ costs (i.e., in an aircraft industry) [2]. Although the probability of some defects is very low for this aviation example, their occurrence has serious consequences for aviation giant such as Boeing and Airbus. This is to say, manufacturers need an integrated strategy to improve the cycle time and the quality of the parts [3].

\footnotetext{
* Corresponding Author.

1 School of Mathematics and Statistics, University of Melbourne, Melbourne, Australia, Email: mehdi.foumani@unimelb.edu.au

${ }^{2}$ School of Industrial Engineering, College of Engineering, University of Tehran, Tehran, Iran, Email: tavakoli@ut.ac.ir
} 
In general, the performance improvement of robotic systems is an interesting research direction (e.g., real world cases [4], evolutionary algorithms for corresponding systems [5] and dynamic robotic systems [6]). The review paper [7] addressed research on the optimization of robotic systems. There is a fairly large number of papers on robotic systems with multiple part types, as two-machine robotic systems with multiple part types are studied on Sethi [8] and Agnetis [9]. There are recent studies on robotic systems with multiple part types, such as Batur et al. [10], Elmi and Topaloglu [11], Batur et al. [12], Gultekin et al. [13]. However, none of them is on robotic systems with controllable times.

There exist only two studies focused on robotic system with controllable times that both are limited to deterministic cases and identical parts production. The first study was stated in Gultekin et al. [14]. They concentrated on the system in which the processing route of parts is unchangeable. Further, there is no non-destructive testing (NDT) in the system, but the processing times of machines can be controllable. Likewise, the outcomes in Gultekin et al. [15] were expanded for a flexible system. In an opposite direction, we cope with stochastic processing route because the test is not destructive, and its required time by robot is controllable. Hence, in addition to considering multiple part types and stochastic cases, the novelty of this paper is that we modify structures of two above studies by considering controllable travel time of the robotic arm that is both material handler and testing device.

The robotic arm here is both material handler and testing device when it transfers a part between machines $[16,17]$. This robotic arm and the corresponding system hereinafter called the MultiFunction Robot (MFR) and the Multi-Function Robotic cell (MFRC), respectively. Foumani et al. $[16,17]$ are restricted to a real-world $M F R$ that only measures the thickness of the identical shaft and records outcomes. However, we here assume the user interface computer is capable of the processing route modification for each part according to its testing result. This is to say that another novelty is that the structures of two above studies is extended for the stochastic situation, as the number of performed NDT tests is random value for each one of parts. Foumani et al. [18] is the single study which implied a practical case of this situation in which a robotic hand of Fanuc M$710 \mathrm{iB} / 45$ Robot has a specific end-effector that test the parts in a way that the number of performed NDT tests is a random value. The robotic hand in the study can cancel transformation by returning to the source machine and loading the defective shaft on it again. However, Foumani et al. [18] is limited to robotic systems with identical parts that is often considered less preferable in the reality. In contrast, the current paper provides details of part sequencing of such MFRCs for the first time in the literature.

As a small-scale $M F R C$, a two-machine $M F R C$ is composed of a manufacturing machine $M_{1}$, a packing machine $M_{2}$, and a $M F R$ that serves the entire system. The part is reloaded on $M_{1}$ for rework if the testing gages of $M F R$ uncovers any parts defects. For a linear layout, the $M F R C$ has separated input hopper $(I)$ and output hopper $(O)$, whereas they are integrated into an input/output hopper $(I / O)$ for a circular layout, or equivalently a cluster tool. Accordingly, we illustrate all parts processing route by $I \rightarrow M F R \rightarrow M_{1 \leftarrow} \rightarrow M F R \rightarrow M_{2} \rightarrow M F R \rightarrow O$ and $I / O \rightarrow M F R \rightarrow M_{1_{\leftarrow}} \rightarrow M F R \rightarrow M_{2}$ $\rightarrow M F R \rightarrow I / O$ for linear and circular layouts. Here, $M_{1}$ and $M F R$ shape the stochastic loop $M_{1} \rightarrow M F R$ in this route.

For $M F R C$ s, the main objective in the literature is to improve their performance, and pickup criterion is one factor that influences the performance. The pickup criterion is classified into three classes: free, no-wait, interval. A free pickup criterion means that a completed part can wait on the machine unlimitedly, whereas it should be unloaded from the machine with no delay and loaded to 
the downstream machine for no-wait pickup criterion. The interval pickup criterion is in-between, such that the machine has a time window for waiting time of the completed part on the machine.

It should be emphasized that the layout is another factor that can influence the performance. Although the processing route of parts is series, $M F R C$ s can have either of linear or circular layouts. A moveable Cartesian robot moves in a rail network for a linear configuration, whereas a stationary base robotic arm rotates on its axis for circular configuration. Note that robotic systems with circular layout are very common in practice, as an especial kind of robotic systems namely cluster tool is using in the semiconductor manufacturing process $[19,20]$.

The final factor that may affect the performance is the robot's travel time between machines that can be additive, Euclidean, and constant. Let us describe three travel time metrics regardless of the multi-functionality of the robotic arm. For additive travel time metric, the robot must pass through all intermediate machines with a fixed speed to move between two machines, whereas the robot's acceleration vary based on the distance between departed and destination machines considering a Euclidean travel time metric. Thus, the Euclidean travel time metric is less time-consuming than the additive one. However, it is not as fast as constant travel time metric where physical distance between all machines is negligible due to the compact size of the system. Many results in the field have been extracted separately for each one of pickup criteria, layouts and travel time metrics, rather than for all of them. We contribute to the literature by studying two-machine $M F R C$ s for the general case.

Since robotic systems with free pickup criterion, circular layout and additive travel time metric are the most prevailing one studied in the literature, the focus of this study is initially on MFRCs with the same characteristics. The rest of this study is as follows. Section 2 includes the problem description. Section 3 is devoted to a concurrent performance analysis of the cost of quality and cycle time for the case of non-identical parts production. Section 4 takes the pickup criterion, layout and travel time metric of the $M F R C$ as a design problem. Finally, Section 5 concludes the research.

\section{Problem description}

For the sake of simplicity, we initially present the standard classification scheme of the problem. The scheme $\omega_{1}\left|\omega_{2}\right| \omega_{3}$ is composed of three fields [21]. $\omega_{1}$ represents the robot and machines characteristics, $\omega_{2}$ stands for the part characteristics, and finally $\omega_{3}$ denotes the objectives. Let us provide details of the classification by some examples. Foumani et al. [18] focused on $R_{M F} C_{2}^{l} \mid($ free, $A$, cyclic -1$) \mid\left(\operatorname{minT}_{1}:\right.$ st. $\left.T_{2}\right)$ that is as follows:

- $\omega_{1}$ : A robotic system with a $M F R$ and two machines that are located in a linear configuration.

- $\omega_{2}$ : Parts have a free pickup criterion, an additive travel time metric, and one unit is completed by execution of a cycle as parts are identical.

- $\omega_{3}$ : The objective is to minimize the expected cycle time given a threshold on the expected cost of quality.

However, we focus on $R_{M F} C_{2}^{O} \mid($ free, $A, M P) \mid \min \lambda T_{1}+(1-\lambda) \cdot T_{2}$ in this section, where the layout is circular, the system processes multiple part types and the objective function has a scalarized formulation of the expected cycle time and cost of quality. Assuming a multiple part scheduling problem, this section is partitioned into three folds: 1) We start with cycle time-related 
definitions. The focus is mainly on $M F R C$ s with free pickup criterion, circular layout and additive travel time metric. 2) The cost of quality-related definitions are mentioned. 3) Definitions related to both time and cost are presented. For production of multiple part types, the system should process a minimal part set (MPS) in a cyclic manner, and therefore each part $l$ is a member of the MPS. The MPS represents the smallest possible set of parts that can fulfill the manufacturing goals [22]. For instance, we assume the supply for four part types should be 100, 60, 80 and 140 units. Then, the greatest common divisor of them is 20, and the quantity of parts in the MPS is 19 (five of the first type, three of the second type, four of the third type and seven of the forth type). This is to say that the dimension of the problem is decreased from $380 ! /(100 ! \times 60 ! \times 80 ! \times 140$ !) to $12 ! /(5 ! \times 3 ! \times 4 ! \times 7 !)$. Also, five parts of the first type are labeled with 1-5, three parts of the second type are labeled with 6-8, four parts of the third type are labeled with 9-12, and finally seven parts of the forth type are labeled with 13-19.

Due to the cyclic behavior of the system, it restarts by processing the first part in the MPS after the last part in the MPS. Then, the period between the completion of exactly same part of two successive MPSs is cycle time in such a mass production. We need to optimize the cycle time by jointly determining the order of $M F R$ activities and the sequence of parts.

Definition 1. A sequence of parts of the MPS is represented by $\sigma$ where $\sigma(l)$ is the $l^{\text {th }}$ part of the corresponding sequence.

It should be noted that, in contrast with the case of identical parts [18], part $l$ here means the part that is located in the $l^{\text {th }}$ position of the corresponding sequence. Since we are focusing on multiple part types, let us break a cycle of the MPS into a set of partial cycles with the size of $n=|\mathrm{MPS}|$. We continue with an updated version of the definition of activity borrowed from Brauner [4].

Definition 2. A robot activity is represented by $A_{i}^{\sigma(l)}, \forall i \in\{0,1,2\}$ and $\forall l \in$ MPS [23], includes steps: 1) $M F R$ takes the part $l$ of sequence $\sigma$ from $M_{i}$. 2) $M F R$ transfers the part to $M_{i+1}$. 3) $M F R$ loads the part onto $M_{i+1}$.

Intuitively, if the system has two machines and a circular layout, $M_{i}$ means $I / O$ for $i=0$ and $M_{i+1}$ means $I / O$ for $i=2$. Additionally, it is vital to stress that Step 2 has an extra segment for $i=1$ due to the fact that the test of part is in transit. We conclude that Step 2 is completed not only if the robotic arm carries the part to the packing machine $M_{2}$, but also the robotic arm shows no fault.

Definition 3. The $l^{\text {th }}$ one-unit partial cycle of the part sequence $\sigma$ is a portion of the cycle where the $l^{\text {th }}$ part of $\sigma$ is the input of the system and the $(l-1)^{\text {th }}$ part of $\sigma$ is the output of the system [23].

Each of these partial cycles can complete a single part. Then, a sequence of robot activities when each activity is performed exactly once for each part $l$, where $l \in$ MPS, represents a partial cycle and $n$ executive partial cycles makes together a cycle. Since the cycle can be broken into $n$ partial cycles, we can find a mechanism to optimize all partial cycles in order to find the optimal cycle. Of course, it is important to find the optimal sequence of parts in order to apply this mechanism for the $M F R C$. Assume that $A_{1}^{\sigma(l)}$ is the final activity for each partial cycle, where $l \in$ MPS. Then, the permutation of other activities can be one of the following alternatives: 1) $A_{2}^{\sigma(l-1)}, A_{0}^{\sigma(l)}$ 2) $A_{0}^{\sigma(l)}$, $A_{2}^{\sigma(l-1)}$. This is to say that we have two classes of one-unit partial cycles: 1) $S_{k 1}^{\sigma(l)}=A_{2}^{\sigma(l-1)}, A_{0}^{\sigma(l)}$, $A_{1}^{\sigma(l)}$ 2) $S_{k 2}^{\sigma(l)}=A_{0}^{\sigma(l)}, A_{2}^{\sigma(l-1)}, A_{1}^{\sigma(l)}$ for the part $l$ entering the system and the part $l-1$ leaving the system. Also, here $k \in\{1,2\}$ is the NDT test strategy. For the stop testing $(k=1) M F R$ stops in front 
of this machine to complete the test, but it performs the test of the part in transit between $M_{1}$ and $M_{2}$ for the transition testing strategy $(k=2)$.

For any $l \in$ MPS, none of $S_{k 1}^{\sigma(l)}$ and $S_{k 2}^{\sigma(l)}$ operate in a steady state due to the dynamic nature of circular MFRCs. Intuitively, the time taken between the completion of $A_{1}^{\sigma(l)}$ and $A_{1}^{\sigma(l+1)}$ is not fixed. Consequently, we present an adapted version of notation:

MPS Minimal part set of a multiple part scheduling problem

$n \quad$ Total number of finished parts in the MPS

$\sigma \quad$ Sequence of parts of the MPS

$\sigma(l) \quad$ Part in the $l^{\text {th }}$ position of the sequence $\sigma$

$\gamma^{\sigma(l)} \quad$ Time required for the NDT test of the $l^{\text {th }}$ part regardless of testing strategy

$\gamma^{L} \quad$ Lower bound of the testing time

$\gamma^{U} \quad$ Upper bound of the testing time

$p_{\sigma(l)} \quad$ Probability of identifying no defect in each time testing of the $l^{\text {th }}$ part

$\varepsilon \quad$ Load/unload time of machines by $M F R$

$\delta \quad$ Time for traveling between adjacent locations with additive travel time metric

$a_{\sigma(l)} \quad$ Processing time of the $l^{\text {th }}$ part by manufacturing machine $M_{1}$

$b_{\sigma(l)} \quad$ Processing time of the $l^{\text {th }}$ part by the packing machine $M_{2}$

$w_{i}^{\sigma(l)} \quad M F R$ 's waiting time at $M_{i}$ for $l^{\text {th }}$ part fed to the $M F R C$, where $l \in \mathbb{N}$

$\bar{a}_{\sigma(l)} \quad$ Bounds for the waiting time $w_{1}^{\sigma(l)}$

$\bar{b}_{\sigma(l)} \quad$ Bounds for the waiting time $w_{2}^{\sigma(l)}$

$f_{1}\left(S_{k j}^{\sigma(l)}, \gamma^{\sigma(l)}\right) \quad$ Total cost of quality for $S_{k j}^{\sigma(l)}$ where $\gamma^{L} \leq \gamma^{\sigma(l)} \leq \gamma^{U}$

$f_{2}\left(S_{k j}^{\sigma(l)}, \gamma^{\sigma(l)}\right) \quad$ Partial cycle time of $S_{k j}^{\sigma(l)}$ where $\gamma^{L} \leq \gamma^{\sigma(l)} \leq \gamma^{U}$

$T_{1} \quad$ Expected cycle time, i.e., $\sum_{l \in M P S} E\left(f_{1}\left(S_{k j}^{\sigma(l)}, \gamma^{\sigma(l)}\right)\right)$

$T_{2} \quad$ Expected cost of quality, i.e., $\sum_{l \in M P S} E\left(f_{2}\left(S_{k j}^{\sigma(l)}, \gamma^{\sigma(l)}\right)\right)$

$\lambda \quad$ Parameter of the scalarization (the weight of the objectives)

$c_{I} \quad$ Total in-situ costs of quality

$c_{E} \quad$ Total out-situ costs of quality

Note that $\varepsilon, \delta, c_{I}$ and $c_{E}$ are assumed to be independent from the part types because the parts in an MPS often belong to the same family $[1,24]$. Here, $\gamma^{\sigma(l)}$ can be varied between the bounds by allocating resources to the NDT. The total cost of resources for each part $l$, where $l \in$ MPS, is dedicated by $f_{1}\left(S_{k j}^{\sigma(l)}, \gamma^{\sigma(l)}\right)$ that is the summation of in-situ and out-situ costs of quality [25]. $c_{I}$ is the cost of NDT to guarantee that all completed parts have a fixed quality level. We may prefer to decrease $c_{I}$. However, it may result in a higher $c_{E}$. 
It is apparent that $f_{1}\left(S_{k j}^{\sigma(l)}, \gamma^{\sigma(l)}\right)$ is non-increasing with respect to $\gamma^{\sigma(l)}$ when $\gamma^{L} \leq \gamma^{\sigma(l)} \leq \gamma^{U}$ while $f_{2}\left(S_{k j}^{\sigma(l)}, \gamma^{\sigma(l)}\right)$ is non-decreasing with respect to $\gamma^{\sigma(l)}$. This is to say, a trade-off between time and cost is vital to know how they vary with respect to the testing time.

Definition 4. For each part $l$ of the part sequence $\sigma$, where $l \in$ MPS, a solution is $\theta^{\sigma(l)}=\left(S^{\sigma(l)}\right.$, $\left.\gamma^{\sigma(l)}\right)$ in which $S^{\sigma(l)} \in\left\{S_{11}^{\sigma(l)}, S_{12}^{\sigma(l)}, S_{21}^{\sigma(l)}, S_{22}^{\sigma(l)}\right\}$ and $\gamma^{L} \leq \gamma^{\sigma(l)} \leq \gamma^{U}$. Moreover, $Y^{\sigma(l)}=\left\{\theta^{\sigma(l)}=\left(S^{\sigma(l)}\right.\right.$, $\left.\gamma^{\sigma(l)}\right): S^{\sigma(l)} \in\left\{S_{11}^{\sigma(l)}, S_{12}^{\sigma(l)}, S_{21}^{\sigma(l)}, S_{22}^{\sigma(l)}\right\}$ and $\left.\gamma^{L} \leq \gamma^{\sigma(l)} \leq \gamma^{U}\right\}$ gives all feasible decisions of the partial cycle executed for the $l^{\text {th }}$ part of a sequence $\sigma$.

Two types of stochastic dominancy exist for optimization problems with two objects: 1) solution $\theta_{1}^{\sigma(l)}$ dominates $\theta_{2}^{\sigma(l)}$ if it is not stochastically worse than $\theta_{2}^{\sigma(l)}$ under both objects, and it is stochastically better than $\theta_{2}^{\sigma(l)}$ under at least one of objects. 2) $\theta_{1}^{\sigma(l)}$ and $\theta_{2}^{\sigma(l)}$ are considered as stochastic alternative solutions if there is no stochastic dominancy between them [14].

Definition 5. If $f_{1}\left(\theta_{1}^{\sigma(l)}\right) \leq f_{1}\left(\theta_{2}^{\sigma(l)}\right)$ and $f_{2}\left(\theta_{1}^{\sigma(l)}\right) \leq f_{2}\left(\theta_{2}^{\sigma(l)}\right)$ with one of the inequalities being strict, then $\theta_{1}^{\sigma(l)}$ stochastically dominates $\theta_{2}^{\sigma(l)}$ for multiple part problem, which is written as $\theta_{1}^{\sigma(l)} \leq \theta_{2}^{\sigma(l)}$.

Definition 6. $S_{k i}^{\sigma(l)}$ stochastically dominates $S_{k j}^{\sigma(l)}\left(S_{k i}^{\sigma(l)} \leq S_{k j}^{\sigma(l)}\right)$ if there exists no $\hat{\gamma} \in \gamma^{*}\left(S_{k j}^{\sigma(l)}\right)$ such that $\left(S_{k j}^{\sigma(l)}, \hat{\gamma}\right) \leq\left(S_{k i}^{\sigma(l)}, \check{\gamma}\right), \forall \check{\gamma} \in \gamma^{*}\left(S_{k i}^{\sigma(l)}\right)$.

Let us now refer to different classes of stochastic dominancy as follows [26]:

1. Absolute dominance $(A D)$ : a random outcome $\Psi_{1}$ is absolute preferred over $\Psi_{2}$, written $\Psi_{1} \geq{ }_{(1)} \Psi_{2}$ if $P\left(\Psi_{1} \geq \Psi_{2}\right)=1$ and $\Psi_{1}>\Psi_{2}$ be met for at least one $\Psi_{1}$.

2. First-order stochastic dominance $(F S D)$ : The random outcome $\Psi_{1}$ is first-order dominant over $\Psi_{2}$, written $\Psi_{1} \geq_{(2)} \Psi_{2}$ if $P\left(\Psi_{1}>\lambda\right) \geq P\left(\Psi_{2}>\lambda\right)$ for all $\lambda$.

3. Second-order stochastic dominance (SSD): if the random outcome $\Psi_{1}$ is second-order dominant over $\Psi_{2}$, written $\Psi_{1} \geq_{(3)} \Psi_{2}$, we conclude that $E\left(\Psi_{1}\right) \geq E\left(\Psi_{2}\right)$.

In a similar manner, $=_{(1)},=_{(2)}$ and $=_{(3)}$ indicate classes of stochastic equalities. $\Psi_{1}={ }_{(1)} \Psi_{2}, \Psi_{1}=_{(2)} \Psi_{2}$ and $\Psi_{1}={ }_{(3)} \Psi_{2}$, if $P\left(\Psi_{1}=\Psi_{2}\right)=1, P\left(\Psi_{1}>\lambda\right)=P\left(\Psi_{2}>\lambda\right)$ and $E\left(\Psi_{1}\right)=E\left(\Psi_{2}\right)$, respectively.

\section{Performance evaluation of objectives}

Before proceeding with this Performance evaluation of objectives, let us first provide a base case formulation of inspired by existing formulations for linear cases [18]. One of the characteristics of $f_{1}\left(S_{k j}^{\sigma(l)}, \gamma^{\sigma(l)}\right)$ is that it is independent of the robotic arm move sequences since it is related to a process-oriented extension of the MFRCs. In more detail, $f_{1}\left(S_{k j}^{\sigma(l)}, \gamma^{\sigma(l)}\right)$ is independent of $\delta$, such that the layout and travel time metric has no effect on it. Accordingly, we formulate $f_{1}\left(S_{k j}^{\sigma(l)}, \gamma^{\sigma(l)}\right)$ for generic two-machine MFRCs as a function of $\gamma^{\sigma(l)}$. For any $l, f_{1}\left(S_{k j}^{\sigma(l)}, \gamma^{\sigma(l)}\right)$ is the summation of the in-situ and out-situ costs. The in-situ cost depends on $\gamma^{\sigma(l)}$ and the probability of identifying no defect. Initially suppose that, for any fixed $\gamma^{\sigma(l)}, M F R$ identifies no defect in each time of test of $l^{\text {th }}$ part with probability $p_{\sigma(l)}$ where $0 \leq p_{\sigma(l)} \leq 1$. This means the testing result of the part $l$ is a 
Bernoulli trail with parameter $p_{\sigma(l)}$. For the $l^{\text {th }}$ part, the treatment of the system is like a geometrically distributed variable $X^{\sigma(l)}$ with parameter $p_{\sigma(l)}$ because the geometric distribution represents a set of Bernoulli trials before the first success. Note that the geometric distribution is memoryless, and therefore we assume that the processing time of each part $l$ on $M_{1}$ is the same for both original and reworking tasks. Similarly, $p_{\sigma(l)}$ is the same for both original and reworking tasks of each part $l$. Hence, the in-situ cost for $l^{\text {th }}$ part is $c_{I} X^{\sigma(l)} \gamma^{\sigma(l)}$. The out-situ cost is only a nonincreasing with respect to $\gamma^{\sigma(l)}$. It is $\frac{c_{E}}{\left(\gamma^{\sigma(l)}\right)^{d}}$ where $d>0$ is a given constant. $d$ is a small value for high-tech industries since even one non-conforming part causes an infinite out-situ cost. The total cost of quality and its expected value for $l^{\text {th }}$ part are:

$$
\begin{array}{r}
f_{1}\left(S_{k j}^{\sigma(l)}, \gamma^{\sigma(l)}\right)=c_{I} X^{\sigma(l)} \gamma^{\sigma(l)}+\frac{c_{E}}{\left(\gamma^{\sigma(l)}\right)^{d}} \\
E\left(f_{1}\left(S_{k j}^{\sigma(l)}, \gamma^{\sigma(l)}\right)\right)=\frac{c_{I} \gamma^{\sigma(l)}}{p_{\sigma(l)}}+\frac{c_{E}}{\left(\gamma^{\sigma(l)}\right)^{d}}
\end{array}
$$

Equation (2) can be generalized to $\sum_{l \in M P S} E\left(f_{1}\left(S_{k j}^{\sigma(l)}, \gamma^{\sigma(l)}\right)\right)=\sum_{l \in M P S} \frac{c_{I} \gamma^{\sigma(l)}}{p_{\sigma(l)}}+\frac{c_{E}}{\left(\gamma^{\sigma(l)}\right)^{d}}$, where $\left(S_{k j}^{\sigma(l)}, \gamma^{\sigma(l)}\right) \in Y^{\sigma(l)}$ if we consider the expected value of total cost of quality for all parts in MPS. Now, we determine feasible partial cycle times for a fixed $\gamma^{\sigma(l)}$. For this case, two strategies exist for the NDT technology: the stop testing $(k=1)$ and the transition testing $(k=2)$. As stated before, for $k=1, M F R$ stops in front of this machine to complete the test, but it performs the test of the part in transit between $M_{1}$ and $M_{2}$ for $k=2$. For a circular two-machine $M F R C$ with additive travel time, the stop testing strategy is restricted to the cases below: 1) it increases the partial cycle time, for at least $\gamma^{\sigma(l)}$ if the testing reveals that the part must pass $\left.M_{1} .2\right)$ it reduces the partial cycle time for at least $\min \left\{\delta, \gamma^{\sigma(l)}\right\}$ if the testing reveals that the part must be loaded to $M_{1}$ for rework. Thus, partial cycle times $S_{11}^{\sigma(l)}, S_{12}^{\sigma(l)}, S_{21}^{\sigma(l)}$ and $S_{22}^{\sigma(l)}$ of a circular two-machine $M F R C$ with additive travel time metric are:

$$
\begin{gathered}
f_{2}\left(S_{11}^{\sigma(l)}, \gamma^{\sigma(l)}\right)=4 \varepsilon+3 \delta+\left(a_{\sigma(l)}+\gamma^{\sigma(l)}+2 \varepsilon\right) X^{\sigma(l)}+b_{\sigma(l-1)} \\
f_{2}\left(S_{12}^{\sigma(l)}, \gamma^{\sigma(l)}\right)=6 \varepsilon+6 \delta+\gamma^{\sigma(l)}+\left(a_{\sigma(l)}+\gamma^{\sigma(l)}+2 \varepsilon\right)\left(X^{\sigma(l)}-1\right)+\max _{a_{\sigma(l)}, b_{\sigma(l-1)}} \\
f_{2}\left(S_{21}^{\sigma(l)}, \gamma^{\sigma(l)}\right)=6 \varepsilon+2 \delta+a_{\sigma(l)}+\max \left\{\delta, \gamma^{\sigma(l)}\right\}+\left(a_{\sigma(l)}+\gamma^{\sigma(l)}+\min \left\{\delta, \gamma^{\sigma(l)}\right\}+2 \varepsilon\right)\left(X^{\sigma(l)}-1\right)+b_{\sigma(l-1)} \\
f_{2}\left(S_{22}^{\sigma(l)}, \gamma^{\sigma(l)}\right)=6 \varepsilon+5 \delta+\max \left\{\delta, \gamma^{\sigma(l)}\right\}+\left(a_{\sigma(l)}+\gamma^{\sigma(l)}+\min \left\{\delta, \gamma^{\sigma(l)}\right\}+2 \varepsilon\right)\left(X^{\sigma(l)}-1\right)+\max _{a_{\sigma(l)}, b_{\sigma(l-1)}}
\end{gathered}
$$

where $\max _{a_{\sigma(l)}, b_{\sigma(l-1)}}=\max \left\{0, a_{\sigma(l)^{-}}(2 \varepsilon+3 \delta), b_{\sigma(l-1)^{-}}(2 \varepsilon+3 \delta)\right\}$. Let us only detail $f_{2}\left(S_{12}^{\sigma(l)}, \gamma^{\sigma(l)}\right)$ for the sake of brevity. In Equation (4), the robotic arm visits $I / O, M_{2}$, and $M_{1}$ in the $l^{\text {th }}$ execution of $S_{12}^{\sigma(l)}$. Firstly, MFR travels backward from $M_{2}$ to $I / O$ to unload the $l^{\text {th }}$ part and load it on $M_{1}(2 \varepsilon+2 \delta)$. Then, it returns to $M_{2}$ to remove the $(l-1)^{\text {th }}$ part and transfer it to $I / O$ after a wait at $M_{2}$ $\left(2 \varepsilon+2 \delta+w_{2}^{\sigma(l-1)}\right)$. Similarly, unoccupied $M F R$ comes back to $M_{1}$ to unload the $l^{\text {th }}$ part and transfer it to $M_{2}$ after a wait at $M_{1}\left(2 \varepsilon+2 \delta+w_{1}^{\sigma(l)}\right)$ in the final step. After testing the part $\left(\gamma^{\sigma(l)}\right)$, it needs the rework time $\left(a_{\sigma(l)}+\gamma^{\sigma(l)}+2 \varepsilon\right)\left(X^{\sigma(l)}-1\right) . \quad$ Thus, $f_{2}\left(S_{12}^{\sigma(l)}\right.$, $\left.\gamma^{\sigma(l)}\right)=6 \varepsilon+6 \delta+\gamma^{\sigma(l)}+\left(a_{\sigma(l)}+\gamma^{\sigma(l)}+2 \varepsilon\right)\left(X^{\sigma(l)}-1\right)+w_{2}^{\sigma(l-1)}+w_{1}^{\sigma(l)}$ where $w_{1}^{\sigma(l)}=\max \left\{0, a_{\sigma(l)}\right.$ 
$\left.(2 \varepsilon+3 \delta)-w_{2}^{\sigma(l-1)}\right\} \quad$ and $\quad w_{2}^{\sigma(l-1)}=\max \left\{0, b_{\sigma(l-1)}-(2 \varepsilon+3 \delta)\right\}$, meaning that $w_{1}^{\sigma(l)}+$ $w_{2}^{\sigma(l-1)}=\max _{a_{\sigma(l)}, b_{\sigma(l-1)}}$.

So far, two frameworks are separately developed. However, MFRCs may face both objectives concurrently. As mentioned earlier, the classification scheme of the problem for this case is $R_{M F} C_{2}^{O} \mid($ free, $A, M P) \mid \min \lambda T_{1}+(1-\lambda) . T_{2}$. Hence, the curve involved in considering both objectives of study provides a high performance with a satisfactory cost. This means the scheduling problem, which is labeled by $T(\sigma)$, is converted into the following weighted sum formulation: $T(\sigma)=\left\{\min \sum_{l \in M P S} \lambda . E\left(f_{1}\left(S_{k j}^{\sigma(l)}, \gamma^{\sigma(l)}\right)\right)+(1-\lambda) . E\left(f_{2}\left(S_{k j}^{\sigma(l)}, \gamma^{\sigma(l)}\right)\right) ;\left(S_{k j}^{\sigma(l)}, \gamma^{\sigma(l)}\right) \in Y^{\sigma(l)}\right\}$. Once the optimal part sequence $\sigma^{*}$ is determined and the optimal partial cycles and corresponding time required for the NDT test of all parts of the MPS are assigned, we can conclude that the value of the optimal solution is $T\left(\sigma^{*}\right)$. The formulation $T(\sigma)=\left\{\min \sum_{l \in M P S} \lambda . E\left(f_{1}\left(S_{k j}^{\sigma(l)}, \gamma^{\sigma(l)}\right)\right)+\right.$ $\left.(1-\lambda) \cdot E\left(f_{2}\left(S_{k j}^{\sigma(l)}, \gamma^{\sigma(l)}\right)\right) ;\left(S_{k j}^{\sigma(l)}, \gamma^{\sigma(l)}\right) \in Y^{\sigma(l)}\right\}$ has $\lambda$ and $1-\lambda$ as the predefined weights for $E\left(f_{1}\left(S_{k j}^{\sigma(l)}, \gamma^{\sigma(l)}\right)\right.$ and $E\left(f_{2}\left(S_{k j}^{\sigma(l)}, \gamma^{\sigma(l)}\right)\right.$, and it can be optimized though the following theorem.

Theorem 1. Table 1 holds for weighted sum formulation of the problem, $T(\sigma)=\left\{\min \sum_{l \in M P S} \lambda . E\left(f_{1}\left(S_{k j}^{\sigma(l)}, \gamma^{\sigma(l)}\right)\right)+(1-\lambda) \cdot E\left(f_{2}\left(S_{k j}^{\sigma(l)}, \gamma^{\sigma(l)}\right)\right) ;\left(S_{k j}^{\sigma(l)}, \gamma^{\sigma(l)}\right) \in Y^{\sigma(l)}\right\}$, if the optimal $\gamma^{\sigma(l)}$ is obtained by first derivative of the weighted sum formulation with respect to $\gamma^{\sigma(l)}$.

Table 1. Optimality region for cycles of circular two-machine $M F R C$ s with non-identical parts

\begin{tabular}{|c|c|c|c|}
\hline \multirow[t]{2}{*}{$\begin{array}{l}\text { Success } \\
\text { Parameter }\end{array}$} & \multicolumn{3}{|c|}{ Operational parameters } \\
\hline & $a_{\sigma(l)}+b_{\sigma(l-1)}<3 \delta$ & $a_{\sigma(l)}+b_{\sigma(l-1)}=3 \delta$ & $a_{\sigma(l)}+b_{\sigma(l-1)}>3 \delta$ \\
\hline$p_{\sigma(l)}<1 / 2$ & $\begin{array}{c}f_{2}\left(S_{11}^{\sigma(l)}, \gamma^{\sigma(l)}\right) \leq_{(1)} f_{2}\left(S_{12}^{\sigma(l)}, \gamma^{\sigma(l)}\right) \\
f_{2}\left(S_{11}^{\sigma(l)}, \gamma^{\sigma(l)}\right) \leq_{(1)} f_{2}\left(S_{21}^{\sigma(l)}, \gamma^{\sigma(l)}\right) \\
f_{2}\left(\theta^{\sigma^{*}(l)}\right)=f_{2}\left(S_{11}^{\sigma(l)}, \gamma^{\sigma(l)}\right)\end{array}$ & $\begin{array}{c}f_{2}\left(S_{11}^{\sigma(l)}, \gamma^{\sigma(l)}\right)={ }_{(1)} f_{2}\left(S_{12}^{\sigma(l)}, \gamma^{\sigma(l)}\right) \\
f_{2}\left(S_{11}^{\sigma(l)}, \gamma^{\sigma(l)}\right) \leq_{(1)} f_{2}\left(S_{21}^{\sigma(l)}, \gamma^{\sigma(l)}\right) \\
f_{2}\left(S_{12}^{\sigma(l)}, \gamma^{\sigma(l)}\right) \leq_{(1)} f_{2}\left(S_{22}^{\sigma(l)}, \gamma^{\sigma(l)}\right) \\
f_{2}\left(\theta^{\sigma^{*}(l)}\right)=f_{2}\left(S_{11}^{\sigma(l)}, \gamma^{\sigma(l)}\right) \& f_{2}\left(S_{12}^{\sigma(l)}, \gamma^{\sigma(l)}\right)\end{array}$ & $\begin{array}{c}f_{2}\left(S_{12}^{\sigma(l)}, \gamma^{\sigma(l)}\right) \leq_{(1)} f_{2}\left(S_{11}^{\sigma(l)}, \gamma^{\sigma(l)}\right) \\
f_{2}\left(S_{12}^{\sigma(l)}, \gamma^{\sigma(l)}\right) \leq_{(1)} f_{2}\left(S_{22}^{\sigma(l)}, \gamma^{\sigma(l)}\right) \\
f_{2}\left(\theta^{\sigma^{*}(l)}\right)=f_{2}\left(S_{12}^{\sigma(l)}, \gamma^{\sigma(l)}\right)\end{array}$ \\
\hline$p_{\sigma(l)}=1 / 2$ & $\begin{array}{c}f_{2}\left(S_{21}^{\sigma(l)}, \gamma^{\sigma(l)}\right)={ }_{(1)} f_{2}\left(S_{11}^{\sigma(l)}, \gamma^{\sigma(l)}\right) \\
f_{2}\left(S_{21}^{\sigma(l)}, \gamma^{\sigma(l)}\right) \leq \leq_{(1)} f_{2}\left(S_{22}^{\sigma(l)}, \gamma^{\sigma(l)}\right) \\
f_{2}\left(S_{11}^{\sigma(l)}, \gamma^{\sigma(l)}\right) \leq{ }_{(1)} f_{2}\left(S_{12}^{\sigma(l)}, \gamma^{\sigma(l)}\right) \\
f_{2}\left(\theta^{\sigma^{*}(l)}\right)=f_{2}\left(S_{11}^{\sigma(l)}, \gamma^{\sigma(l)}\right) \\
\quad \& f_{2}\left(S_{21}^{\sigma(l)}, \gamma^{\sigma(l)}\right)\end{array}$ & $\begin{array}{c}f_{2}\left(S_{11}^{\sigma(l)}, \gamma^{\sigma(l)}\right)={ }_{(1)} f_{2}\left(S_{12}^{\sigma(l)}, \gamma^{\sigma(l)}\right)={ }_{(1)} f_{2}\left(S_{22}^{\sigma(l)}, \gamma^{\sigma(l)}\right) \\
={ }_{(1)} f_{2}\left(S_{21}^{\sigma(l)}, \gamma^{\sigma(l)}\right) \\
f_{2}\left(\theta^{\sigma^{*}(l)}\right)=f_{2}\left(S_{11}^{\sigma(l)}, \gamma^{\sigma(l)}\right) \& f_{2}\left(S_{21}^{\sigma(l)}, \gamma^{\sigma(l)}\right) \\
\& f_{2}\left(S_{12}^{\sigma(l)}, \gamma^{\sigma(l)}\right) \& f_{2}\left(S_{22}^{\sigma(l)}, \gamma^{\sigma(l)}\right)\end{array}$ & $\begin{array}{c}f_{2}\left(S_{12}^{\sigma(l)}, \gamma^{\sigma(l)}\right)={ }_{(1)} f_{2}\left(S_{22}^{\sigma(l)}, \gamma^{\sigma(l)}\right) \\
f_{2}\left(S_{12}^{\sigma(l)}, \gamma^{\sigma(l)}\right) \leq{ }_{(1)} f_{2}\left(S_{11}^{\sigma(l)}, \gamma^{\sigma(l)}\right) \\
f_{2}\left(S_{22}^{\sigma(l)}, \gamma^{\sigma(l)}\right) \leq{ }_{(1)} f_{2}\left(S_{21}^{\sigma(l)}, \gamma^{\sigma(l)}\right) \\
f_{2}\left(\theta^{\sigma^{*}(l)}\right)=f_{2}\left(S_{12}^{\sigma(l)}, \gamma^{\sigma(l)}\right) \\
\quad \& f_{2}\left(S_{22}^{\sigma(l)}, \gamma^{\sigma(l)}\right)\end{array}$ \\
\hline$p_{\sigma(l)}>1 / 2$ & $\begin{array}{c}f_{2}\left(S_{21}^{\sigma(l)}, \gamma^{\sigma(l)}\right) \leq{ }_{(1)} f_{2}\left(S_{11}^{\sigma(l)}, \gamma^{\sigma(l)}\right) \\
f_{2}\left(S_{21}^{\sigma(l)}, \gamma^{\sigma(l)}\right) \leq{ }_{(1)} f_{2}\left(S_{22}^{\sigma(l)}, \gamma^{\sigma(l)}\right) \\
f_{2}\left(\theta^{\sigma^{*}(l)}\right)=f_{2}\left(S_{21}^{\sigma(l)}, \gamma^{\sigma(l)}\right)\end{array}$ & $\begin{array}{c}f_{2}\left(S_{22}^{\sigma(l)}, \gamma^{\sigma(l)}\right)={ }_{(1)} f_{2}\left(S_{21}^{\sigma(l)}, \gamma^{\sigma(l)}\right) \\
f_{2}\left(S_{22}^{\sigma(l)}, \gamma^{\sigma(l)}\right) \leq_{(1)} f_{2}\left(S_{12}^{\sigma(l)}, \gamma^{\sigma(l)}\right) \\
f_{2}\left(S_{21}^{\sigma(l)}, \gamma^{\sigma(l)}\right) \leq_{(1)} f_{2}\left(S_{11}^{\sigma(l)}, \gamma^{\sigma(l)}\right) \\
f_{2}\left(\theta^{\sigma^{*}(l)}\right)=f_{2}\left(S_{21}^{\sigma(l)}, \gamma^{\sigma(l)}\right) \& f_{2}\left(S_{22}^{\sigma(l)}, \gamma^{\sigma(l)}\right)\end{array}$ & $\begin{array}{c}f_{2}\left(S_{22}^{\sigma(l)}, \gamma^{\sigma(l)}\right) \leq_{(1)} f_{2}\left(S_{21}^{\sigma(l)}, \gamma^{\sigma(l)}\right) \\
f_{2}\left(S_{22}^{\sigma(l)}, \gamma^{\sigma(l)}\right) \leq_{(1)} f_{2}\left(S_{12}^{\sigma(l)}, \gamma^{\sigma(l)}\right) \\
f_{2}\left(\theta^{\sigma^{*}(l)}\right)=f_{2}\left(S_{22}^{\sigma(l)}, \gamma^{\sigma(l)}\right)\end{array}$ \\
\hline
\end{tabular}

Proof. The idea behind the proof of this theorem is as follows. Recalling the generalized version of Equation (2), the chosen partial cycle has no effect on the value of $\sum_{l \in M P S} E\left(f_{1}\left(S_{k j}^{\sigma(l)}, \gamma^{\sigma(l)}\right)\right)$. Therefore, $\sum_{l \in M P S} \lambda . E\left(f_{1}\left(S_{k j}^{\sigma(l)}, \gamma^{\sigma(l)}\right)\right)$ plays the role of an intercept when we select the optimal among $S_{11}^{\sigma(l)}, S_{12}^{\sigma(l)}, S_{21}^{\sigma(l)}$ and $S_{22}^{\sigma(l)}$ for a part. This is because Table 1 implies $a_{\sigma(l)}, b_{\sigma(l)}$, $\delta$ and $p_{\sigma(l)}$ affect the optimality of a partial cycle, not $\gamma^{\sigma(l)}$. Hence, the segment 
$\sum_{l \in M P S} \lambda . E\left(f_{1}\left(S_{k j}^{\sigma(l)}, \gamma^{\sigma(l)}\right)\right)$ is the same for all parts, and can be ignored when we select the optimal cycles.

Regarding Table 1, let us only focus on one part and prove the optimality region $z^{\sigma^{*}(l)}=\left(E\left(f_{1}\left(S_{11}^{\sigma(l)}\right.\right.\right.$, $\left.\gamma^{*}\left(S_{11}^{\sigma(l)}\right)\right), E\left(f_{2}\left(S_{11}^{\sigma(l)}, \gamma^{*}\left(S_{11}^{\sigma(l)}\right)\right)\right)$; the proof of the other bounds in Table 1 has symmetry. Having Definition $6, S_{11}^{\sigma(l)}$ dominates another cycles if there is no $\hat{\gamma}_{k j} \in \gamma^{*}\left(S_{k j}^{\sigma(l)}\right)$ so that $\left(S_{k j}^{\sigma(l)}, \hat{\gamma}\right) \leq\left(S_{11}^{\sigma(l)}\right.$, $\check{\gamma}), \forall \check{\gamma} \in \gamma^{*}\left(S_{11}^{\sigma(l)}\right)$ and $\forall S_{k j}^{\sigma(l)} \in\left\{S_{12}^{\sigma(l)}, S_{21}^{\sigma(l)}, S_{22}^{\sigma(l)}\right\}$. Then, considering a constant $\mu$, we have:

$$
\begin{gathered}
E\left(f_{2}\left(S_{11}^{\sigma(l)}, \breve{\gamma}\right)\right)=\mu \leftrightarrow 4 \varepsilon+3 \delta+\frac{a_{\sigma(l)}+\check{\gamma}+2 \varepsilon}{p_{\sigma(l)}}+b_{\sigma(l-1)}=\mu \\
E\left(f_{2}\left(S_{12}^{\sigma(l)}, \hat{\gamma}_{12}\right)\right)=\mu \leftrightarrow 4 \varepsilon+6 \delta-a_{\sigma(l)}+\frac{a_{\sigma(l)}+\widehat{\gamma}_{12}+2 \varepsilon}{p_{\sigma(l)}}+\max _{a_{\sigma(l)}, b_{\sigma(l-1)}}=\mu \\
E\left(f_{2}\left(S_{21}^{\sigma(l)}, \hat{\gamma}_{21}\right)\right)=\mu \leftrightarrow 4 \varepsilon+2 \delta+\max \left\{\delta, \hat{\gamma}_{21}\right\}-\hat{\gamma}_{21}-\min \left\{\delta, \hat{\gamma}_{21}\right\}+\frac{a_{\sigma(l)}+\widehat{\gamma}_{21}+\min \left\{\delta, \widehat{\gamma}_{21}\right\}+2 \varepsilon}{p_{\sigma(l)}}+b_{\sigma(l-1)}=\mu \\
E\left(f_{2}\left(S_{22}^{\sigma(l)}, \hat{\gamma}_{22}\right)\right)=\mu \leftrightarrow 4 \varepsilon+5 \delta+\max \left\{\delta, \hat{\gamma}_{22}\right\}-\hat{\gamma}_{22}-\min \left\{\delta, \hat{\gamma}_{22}\right\}-a_{\sigma(l)}+\frac{a_{\sigma(l)}+\widehat{\gamma}_{22}+\min \left\{\delta, \hat{\gamma}_{22}\right\}+2 \varepsilon}{p_{\sigma(l)}}+\max _{a_{\sigma(l)}, b_{\sigma(l-1)}}=\mu
\end{gathered}
$$

Which meaning that:

$$
\begin{gathered}
\check{\gamma}=p_{\sigma(l)}\left(\mu-4 \varepsilon-3 \delta-b_{\sigma(l)}\right)-a_{\sigma(l)}-2 \varepsilon \\
\hat{\gamma}_{12}=p_{\sigma(l)}\left(\mu+a_{\sigma(l)}-4 \varepsilon-6 \delta-\max _{a_{\sigma(l)}, b_{\sigma(l-1)}}\right)-a_{\sigma(l)}-2 \varepsilon \\
\hat{\gamma}_{21}=p_{\sigma(l)}\left(\mu+\hat{\gamma}_{21}+\min \left\{\delta, \hat{\gamma}_{21}\right\}-4 \varepsilon-2 \delta-\max \left\{\delta, \hat{\gamma}_{21}\right\}-b_{\sigma(l-1)}\right)-a_{\sigma(l)}-\min \left\{\delta, \hat{\gamma}_{21}\right\}-2 \varepsilon \\
\hat{\gamma}_{22}=p_{\sigma(l)}\left(\mu+\hat{\gamma}_{22}+a_{\sigma(l)}+\min \left\{\delta, \hat{\gamma}_{22}\right\}-4 \varepsilon-5 \delta-\max \left\{\delta, \hat{\gamma}_{22}\right\}-\max a_{\sigma(l)}, b_{\sigma(l-1)}\right)-a_{\sigma(l)}-\min \left\{\delta, \hat{\gamma}_{22}\right\}-2 \varepsilon
\end{gathered}
$$

Taking $\mu$ from (7) and combining it with (12), (13) and (14) results in:

$$
\begin{gathered}
\hat{\gamma}_{12}=\check{\gamma}+p_{\sigma(l)}\left(a_{\sigma(l)}+b_{\sigma(l)}-3 \delta-\max a_{a_{\sigma(l)}, b_{\sigma(l-1)}}\right) \\
\hat{\gamma}_{21}=\check{\gamma}+2 p_{\sigma(l)}\left(\min \left\{\delta, \hat{\gamma}_{21}\right\}\right)-\min \left\{\delta, \hat{\gamma}_{21}\right\} \\
\hat{\gamma}_{22}=\check{\gamma}+p_{\sigma(l)}\left(a_{\sigma(l)}+b_{\sigma(l)}+\hat{\gamma}_{22}+\min \left\{\delta, \hat{\gamma}_{22}\right\}-2 \delta-\max \left\{\delta, \hat{\gamma}_{22}\right\}-\max a_{a_{\sigma(l)}, b_{\sigma(l-1)}}\right)-\min \left\{\delta, \hat{\gamma}_{22}\right\}
\end{gathered}
$$

It follows form (15) and (16) that $\hat{\gamma}_{12}<\check{\gamma}$ and $\hat{\gamma}_{21}<\check{\gamma}$ if $a_{\sigma(l)}+b_{\sigma(l-1)}<3 \delta$ and $p_{\sigma(l)}<1 / 2$. Moreover, (17) reveals that $\hat{\gamma}_{22}<\check{\gamma}$ is correct if $a_{\sigma(l)}+b_{\sigma(l-1)}<3 \delta$ and $p_{\sigma(l)}<1 / 2$. Then, $S_{11}^{\sigma(l)} \leq S_{12}^{\sigma(l)}, S_{11}^{\sigma(l)} \leq S_{21}^{\sigma(l)}$, $S_{11}^{\sigma(l)} \leq S_{22}^{\sigma(l)}$ and $z^{\sigma^{*}(l)}=\left(E\left(f_{1}\left(S_{11}^{\sigma(l)}, \gamma^{*}\left(S_{11}^{\sigma(l)}\right)\right), E\left(f_{2}\left(S_{11}^{\sigma(l)}, \gamma^{*}\left(S_{11}^{\sigma(l)}\right)\right)\right)\right.\right.$. The proof of Table 1 for the rest of partial cycles is similar if we limit ourselves to a single part $l$ of MPS. It is obvious that we should sum individual $E\left(f_{2}\left(S_{k j}^{\sigma(l)}, \gamma^{\sigma(l)}\right)\right)$ in order to obtain $\sum_{l \in M P S}(1-\lambda) . E\left(f_{2}\left(S_{k j}^{\sigma(l)}, \gamma^{\sigma(l)}\right)\right)$.

We could prove that $S_{k j}^{\sigma(l)}$ should be chosen based on Table 1. After selection of the optimal partial cycles for all parts in MPS, the question is: what is the optimal value of $\gamma^{\sigma(l)}$ afterwards? Here, we 
are not able to simply consider the first derivative of Equation (2) equal to zero $\left(\frac{d}{d \gamma}\left(\frac{c_{I} \gamma^{\sigma(l)}}{p_{\sigma(l)}}\right.\right.$ $\left.\left.+\frac{c_{E}}{\left(\gamma^{\sigma(l)}\right)^{d}}\right)=0\right)$ to obtain the optimal $\gamma^{\sigma(l)}$. Instead, we should consider a generalized form as $\frac{d}{d \gamma}$ $\left(\lambda \cdot\left(\frac{c_{I} \gamma^{\sigma(l)}}{p_{\sigma(l)}}+\frac{c_{E}}{\left(\gamma^{\sigma(l)}\right)}\right)+(1-\lambda) \cdot E\left(f_{2}\left(S_{k j}^{\sigma(l)}, \gamma^{\sigma(l)}\right)\right)\right)=0$ for each part $l$ given that the optimal partial cycle is obtained from Table 1. For instance, if Table 1 implies that $S_{11}^{\sigma(l)}$ is the optimal partial cycle $\left(a_{\sigma(l)}+b_{\sigma(l-1)}<3 \delta\right.$ and $\left.p_{\sigma(l)}<1 / 2\right)$, then $\gamma^{*}\left(S_{k j}^{\sigma(l)}\right)=\sqrt[d+1]{\frac{d \cdot p_{\sigma(l)} \cdot c_{E}}{1+c_{I}}} \square$.

Corollary 1. Having a circular two-machine $M F R C$ with additive travel time metric that produces non-identical parts, the problem of finding the optimal part sequence $\sigma^{*}$ is equivalent to the travelling salesman problem (TSP) if testing result on MFR is Bernoulli trail for all parts.

The idea behind the proof of Corollary 1 is straightforward. We know that Table 1 plays the role of a set of dominant rules as mentioned before. Thus, for each part sequence $\sigma$, we can simply find the optimal partial cycles and corresponding time required for the NDT test of all parts of the MPS. All we need is to find the optimal part sequence $\sigma^{*}$. Regardless of the applied part sequence, this is equivalent to a TSP with cost matrix $C=\left[E\left(f_{2}\left(S_{k j}^{l}, \gamma\right)\right]_{n \times n}\right.$ due to the following reason. Recalling Definition 3, the cost matrix shows part $l-1$ (as output of the partial cycle) on the row and part $l$ (as input of the partial cycle) on the column. Hence, the obtained $\sigma$ by this cost matrix can give us $\sigma^{*}$.

The cost matrix plays the role of a prerequisite for converting the problem into a TSP problem, such that we can find the optimal sequence of parts with TSP solvers such as Concorde [27]. It is well known that TSP is an NP-hard problem in combinatorial optimization, and therefore solving its graph is computationally complex. However, the class of TSP which is equivalent to our robotic system problem can be solved in polynomial-time due to the structure of the cost matrix. Regardless of the NDT, the well-known Gilmore and Gomory (GG) algorithm is applied for typical robotic systems with non-identical parts [28]. However, in the following theorem, we show how a GG algorithm can be employed to find optimal sequence of a two-machine MFRC with NDT assumption.

Theorem 2. The class of the TSP that is equivalent to the two-machine MFRCs with non-identical parts is solvable in polynomial time.

Proof. The idea behind the proof of this theorem is as follows. The GG algorithm solves this special case of the TSP with cost structure $C_{i j}=\max \left\{x_{l}, y_{m}\right\}$. To describe the GG algorithm, we should first rewrite the expected values of Equations (3)-(6). The expected values of Equations (3) and (5) are:

$$
\begin{gathered}
\sum_{l \in M P S} E\left(f_{2}\left(S_{11}^{\sigma(l)}, \gamma^{\sigma(l)}\right)\right)=n \alpha_{1}+\sum_{l=1}^{n} \frac{a_{\sigma(l)}+\gamma^{\sigma(l)}+2 \varepsilon}{p_{\sigma(l)}}+\sum_{l=1}^{n} b_{\sigma(l-1)} \\
\sum_{l \in M P S} E\left(f_{2}\left(S_{21}^{\sigma(l)}, \gamma^{\sigma(l)}\right)\right)=n \alpha_{3}+\sum_{l=1}^{n}\left(\max \left\{\delta, \gamma^{\sigma(l)}\right\}-\gamma^{\sigma(l)}-\min \left\{\delta, \gamma^{\sigma(l)}\right\}+\frac{a_{\sigma(l)}+\gamma^{\sigma(l)}+\min \left\{\delta, \gamma^{\sigma(l)}\right\}+2 \varepsilon}{p_{\sigma(l)}}\right)+\sum_{l=1}^{n} b_{\sigma(l-1)}
\end{gathered}
$$


, where $\alpha_{1}=4 \varepsilon+3 \delta$ and $\alpha_{3}=4 \varepsilon+2 \delta$. It is obvious that $\sum_{l \in M P S} E\left(f_{2}\left(S_{11}^{\sigma(l)}, \gamma^{\sigma(l)}\right)\right)$ and $\sum_{l \in M P S} E\left(f_{2}\left(S_{21}^{\sigma(l)}, \gamma^{\sigma(l)}\right)\right)$ are independent from the sequence of parts in the MPS. This means that there is no part sequensing problem if each part $l$ is processed by either of $S_{11}^{\sigma(l)}$ or $S_{21}^{\sigma(l)}$.

$$
\begin{aligned}
& \sum_{l \in M P S} E\left(f_{2}\left(S_{12}^{\sigma(l)}, \gamma^{\sigma(l)}\right)\right)=n \alpha_{2}+\sum_{l=1}^{n}\left(\frac{a_{\sigma(l)}+\gamma^{\sigma(l)}+2 \varepsilon}{p_{\sigma(l)}}-a_{\sigma(l)}\right)+\sum_{l=1}^{n} \max _{a_{\sigma(l)}, b_{\sigma(l-1)}} \\
& \sum_{l \in M P S} E\left(f_{2}\left(S_{22}^{\sigma(l)}, \gamma^{\sigma(l)}\right)\right)=n \alpha_{4} \\
& +\sum_{l=1}^{n}\left(\max \left\{\delta, \gamma^{\sigma(l)}\right\}-\gamma^{\sigma(l)}-\min \left\{\delta, \gamma^{\sigma(l)}\right\}-a_{\sigma(l)}+\frac{a_{\sigma(l)}+\gamma^{\sigma(l)}+\min \left\{\delta, \gamma^{\sigma(l)}\right\}+2 \varepsilon}{p_{\sigma(l)}}\right) \\
& +\sum_{l=1}^{l} \max _{a_{\sigma(l)}, b_{\sigma(l-1)}}
\end{aligned}
$$

, where $\alpha_{2}=4 \varepsilon+6 \delta$ and $\alpha_{4}=4 \varepsilon+5 \delta$. Once again, it is obvious that $\alpha_{2}, \alpha_{4}$, first summations in Equations (20) and (21) are all independent from the sequence of parts in the MPS because they show no interaction between two consequtive parts in an arbitrary sequence $\sigma$. However, the second summations in Equations (20) and (21), which are $\sum_{l=1}^{n} \max _{a_{\sigma(l)}, b_{\sigma(l-1)}}$, depend on the parts sequence $\sigma$. Consequently, we need to detail $\max _{a_{\sigma(l)}, b_{\sigma(l-1)}}=\max \left\{0, a_{\sigma(l)^{-}}(2 \varepsilon+3 \delta), b_{\sigma(l-1)^{-}}\right.$ $(2 \varepsilon+3 \delta)\}$.

As mentioned in the proof of Equations (3)-(6), $\max _{a_{\sigma(l)}, b_{\sigma(l-1)}}=w_{1}^{\sigma(l)}+w_{2}^{\sigma(l-1)}$ where $w_{1}^{\sigma(l)}=$ $\max \left\{0, a_{l}-(2 \varepsilon+3 \delta)-w_{2}^{\sigma(l-1)}\right\}$ and $w_{2}^{\sigma(l-1)}=\max \left\{0, b_{\sigma(l-1)}-(2 \varepsilon+3 \delta)\right\}$. Here, $w_{2}^{\sigma(l-1)}$ is independent from the part sequence $\sigma$, but the part sequence has a direct effect on $w_{1}^{\sigma(l)}$ and this is the reason of dependency of $\max _{a_{\sigma(l)}, b_{\sigma(l-1)}}$ to the part sequence $\sigma$. Let us write $w_{1}^{\sigma(l)}$ as $w_{1}^{\sigma(l)}=$ $-w_{2}^{\sigma(l-1)}+\max \left\{w_{2}^{\sigma(l-1)}, a_{\sigma(l)}-(2 \varepsilon+3 \delta)\right\}$. Then, Equations (20-21) are changed as follows:

$$
\begin{aligned}
\sum_{l \in M P S} E & \left(f_{2}\left(S_{12}^{\sigma(l)}, \gamma^{\sigma(l)}\right)\right)=n \alpha_{5}+\sum_{l=1}^{n}\left(\frac{a_{\sigma(l)}+\gamma^{\sigma(l)}+2 \varepsilon}{p_{\sigma(l)}}-a_{\sigma(l)}\right)+\sum_{l=1}^{n} \max \left\{x_{\sigma(l-1)}, y_{\sigma(l)}\right\} \\
& \sum_{l \in M P S} E\left(f_{2}\left(S_{22}^{\sigma(l)}, \gamma^{\sigma(l)}\right)\right)=n \alpha_{6} \\
& +\sum_{\substack{l=1 \\
n}}\left(\max \left\{\delta, \gamma^{\sigma(l)}\right\}-\gamma^{\sigma(l)}-\min \left\{\delta, \gamma^{\sigma(l)}\right\}-a_{\sigma(l)}+\frac{a_{\sigma(l)}+\gamma^{\sigma(l)}+\min \left\{\delta, \gamma^{\sigma(l)}\right\}+2 \varepsilon}{p_{\sigma(l)}}\right) \\
& +\sum_{l=1}^{n} \max \left\{x_{\sigma(l-1)}, y_{\sigma(l)}\right\}
\end{aligned}
$$

, where $\alpha_{5}=2 \varepsilon+3 \delta, \alpha_{6}=2 \varepsilon+2 \delta, x_{\sigma(l-1)}=\max \left\{2 \varepsilon+3 \delta, b_{\sigma(l-1)}\right\}, y_{\sigma(l)}=a_{l}$. In these equations, the only segment that is affected by the parts sequence is $\sum_{l=1}^{n} \max \left\{x_{\sigma(l-1)}, y_{\sigma(l)}\right\}$, which has the structure of a polynomial-time solvable TSP, and a GG algorithm can be implemented for it $\square$.

For the sake of brevity, we refer to Kabadi and Fazle Baki [28] to know how the above GG algorithm can be implemented for two-machine $M F R C$ s in polynomial time. So far, there is a unified framework for two-machines $M F R C$ s for non-identical parts production with following 
characteristics: free pickup criterion, circular layout and additive travel time metric. The framework assists companies which use robots with multi-functionality to remain competitive. However, a generalization of the framework is useful because types of robotic systems are varied by certain features. In the next section, we start by providing a generalization of the framework that distinguishes the problem based on three characteristics of MFRCs: pickup criterion, layout and travel time metric.

\section{MFRCs based on pickup criterion, layout and travel time metric}

This research has concentrated on typical MFRCs to validate the study of the case of nonidentical parts. Clearly, it is still crucial to show that the analysis is adoptable to real-world cases, which are common in different industries. The applicability of the analysis is indicated by three main characteristics of MFRCs. The first characteristic is described separately, but the last two characteristics are described jointly in order to conduct a more in-depth analysis.

\subsection{Analysis of MFRCs based on pickup criterion}

There is a meaningful interaction between optimal solution of production systems and their features. Thus, here, we study problems with the standard classification schemes $R_{M F} C_{2}^{O} \mid$ (no wait, $A, M P) \mid \min \lambda T_{1}+(1-\lambda) \cdot T_{2} \quad$ and $\quad R_{M F} C_{2}^{O} \mid($ interval, $A, M P) \mid \min \lambda T_{1}+(1-\lambda) . T_{2}$, respectively. The former scheme represents a robotic system with a MFR and two machines that are located in a circular configuration. Multiple part types have a no-wait pickup criterion with an additive travel time metric, and the objective has a scalarized formulation. However, the latter scheme represents a similar robotic system where pickup criterion is interval. The no-wait and interval pickup criteria are more oriented towards real world problems in comparison with free pickup criterion since it is a relaxed version of them. In practice, the no-wait and interval pickup criteria are applied for chemical and food products that should be processed as soon as possible. For no-wait pickup criterion, the waiting time of each part $l$ on each one of machines $M_{1}$ and $M_{2}$ is zero, whereas the waiting time of each part $l$ on each one of $M_{1}$ and $M_{2}$ is limited within a time interval for the interval pickup criterion.

As stated in Foumani et al. [29], the difference between MFRCs with free pickup criterion and two others lies in the fact that we need to define optimality conditions for MFRCs with free pickup criterion. However, we must find feasibility conditions for each of $S_{k j}^{\sigma(l)}$ in the cases of no-wait and interval pickup criteria. To point out the subject more clearly, let us first determine the feasibility conditions for MFRCs with no-wait pickup criterion.

$$
\begin{aligned}
a_{\sigma(l)} & \geq 2 \varepsilon+3 \delta \\
b_{\sigma(l-1)} & \geq 2 \varepsilon+3 \delta
\end{aligned}
$$

Inequalities (24) and (25) imply that $w_{1}^{\sigma(l)}$ and $w_{2}^{\sigma(l-1)}$ should be qual to zero in order to have a feasible partial cycle for $l^{\text {th }}$ part in the MPS. It can be argued that partial cycles $S_{11}^{\sigma(l)}$ and $S_{21}^{\sigma(l)}$ are feasible, regardless of $a_{\sigma(l)}$ and $b_{\sigma(l-1)}$ in inequalities (24) and (25), but $S_{12}^{\sigma(l)}$ and $S_{22}^{\sigma(l)}$ are more productive when they are feasible. This is because $w_{1}^{\sigma(l)}$ and $w_{2}^{\sigma(l-1)}$ are zero, meaning that 
$\max _{a_{\sigma(l)}, b_{\sigma(l-1)}}=0$ in Equations (4) and (6). Therefore, Equations (4) and (6) dominate Equations (3) and (5) with the same value of the total cost of quality. After these feasibility checks, Theorems 1 and 2 can be applied for MFRCs with no-wait pickup criterion. In what follows, we determine the feasibility conditions for $M F R C$ s with interval pickup criterion.

$$
\begin{aligned}
a_{\sigma(l)}+\bar{a}_{\sigma(l)} & \geq 2 \varepsilon+3 \delta \\
b_{\sigma(l-1)}+\bar{b}_{\sigma(l-1)} & \geq 2 \varepsilon+3 \delta
\end{aligned}
$$

, where $\bar{a}_{\sigma(l)}$ and $\bar{b}_{\sigma(l-1)}$ denote the bounds for the waiting times $w_{1}^{\sigma(l)}$ and $w_{2}^{\sigma(l-1)}$, respectively. Because waiting times $w_{1}^{\sigma(l)}$ and $w_{2}^{\sigma(l-1)}$ can be nonzero, $S_{12}^{\sigma(l)}$ and $S_{22}^{\sigma(l)}$ are not necessarily optimal even if they are feasibly. Table 1 is for optimally check in addition to feasibility inequalities (26) and (27).

\subsection{Analysis of MFRCs based on layout and travel time metric}

At this stage, we consider replacing the layout of the $M F R C$ with a linear configuration and changing its travel time metric to either of Euclidean and constant to know how this feature impacts the efficiency. Therefore, in this section, we study problems with following classification schemes:

- $\quad R_{M F} C_{2}^{l} \mid($ free, $A, M P) \mid \min \lambda T_{1}+(1-\lambda) \cdot T_{2}$

- $\quad R_{M F} C_{2}^{O} \mid($ free, $E, M P) \mid \min \lambda T_{1}+(1-\lambda) \cdot T_{2}$

- $\quad R_{M F} C_{2}^{O} \mid($ free, $C, M P) \mid \min \lambda T_{1}+(1-\lambda) . T_{2}$

The difference of the first classification scheme with the classification scheme in Section 2 is that it represents a linear configuration, whereas the difference of the last two classification schemes with the classification scheme in Section 2 is that they represent Euclidean and constant travel time metrics. We first derive the travel time of the $M F R$ between machines for $m$-machine $M F R C$ with any layout and travel time metric, and then extract some special properties of the two-machine cases. We recall that $\delta$ represents the required time for traveling between two adjacent location pairs, i.e., $e$ and $e+1$. That is, the travel time $d\left(M_{\mathrm{e}}, M_{\mathrm{f}}\right)$ between two non-adjacent location pairs $e$ and $f$ for each one of two layouts and three travel time metrics can be expressed in Table 2 (inspired from Jolai et al. [30]).

Let us start with linear and circular MFRCs with additive travel time metric. Without loss of generality, assume that $e<f$. Then, as stated earlier, the linear case has only one route $M_{\mathrm{e}} \rightarrow M_{\mathrm{e}+1} \rightarrow \ldots \rightarrow M_{\mathrm{f}-1} \rightarrow M_{\mathrm{f}}$, to transfer the part from $M_{\mathrm{e}}$ to $M_{\mathrm{f}}$, whilst there are two options $M_{\mathrm{e}} \rightarrow M_{\mathrm{e}+1} \rightarrow \ldots \rightarrow M_{\mathrm{f}-1} \rightarrow M_{\mathrm{f}}$ and $M_{\mathrm{e}} \rightarrow M_{\mathrm{e}-1} \rightarrow \ldots \rightarrow I / O \rightarrow \ldots \rightarrow M_{\mathrm{f}+1} \rightarrow M_{\mathrm{f}}$ possible for the circular case to travel between $M_{\mathrm{e}}$ to $M_{\mathrm{f}}$ and these two routes can potentially give a shorter travel time. Generally, the time taken for a move from $M_{\mathrm{e}}$ to $M_{\mathrm{f}}$ is $|e-f| \delta$ and $\min \{|e-f| \delta,(m+1-|e-f|) \delta\}$ in linear and circular configurations of MFRCs. Because $|e-f| \delta \geq \min \{|e-f| \delta,(m+1-|e-f|) \delta\}$, the travel time between machines $M_{\mathrm{e}}$ to $M_{\mathrm{f}}$ in the linear case of $M F R C \mathrm{~s}$ is never less than this value in the circular case of $M F R C$ s. For the case of Euclidean travel time metric, the $M F R$ s acceleration and deceleration vary based on the distance between machines $M_{\mathrm{e}}$ and $M_{\mathrm{f}}$. Therefore, a direct move between them without stop takes $\delta_{\text {ef }}$ and it is shorter than the time $\delta_{\text {eg }}+\delta_{\mathrm{gf}}$ taken for a move with stop. Likewise, possibility of two options $M_{\mathrm{e}} \rightarrow M_{\mathrm{e}+1} \rightarrow \ldots \rightarrow M_{\mathrm{f}-1} \rightarrow M_{\mathrm{f}}$ and $M_{\mathrm{e}} \rightarrow M_{\mathrm{e}-1} \rightarrow \ldots \rightarrow I / O \rightarrow \ldots \rightarrow M_{\mathrm{f}+1} \rightarrow M_{\mathrm{f}}$ of routing in MFRCs with Euclidean travel time metric results in time savings. However, this result does not hold 
for the constant travel time metric. In more detail, we know that additive travel time metric is more time-consuming than Euclidean travel time metric $\left(\delta_{\mathrm{ef}}=\delta_{\mathrm{eg}}+\delta_{\mathrm{gf}}\right)$, while the constant travel time metric reflects the ultimate gain of Euclidean travel time metric $\left(\delta_{\mathrm{ef}}=\delta\right)$. From this discussion, we can conclude that two options explained above take the travel time $\delta$ that is independent from the layout of the MFRCs. The results of Table 2 are especially suitable for the optimization of twomachine MFRCs due to their structural properties. Table 3 is provided for $S_{11}^{\sigma(l)}, S_{12}^{\sigma(l)}, S_{21}^{\sigma(l)}$ and $S_{22}^{\sigma(l)}$.

Table 2. Travel time of the $M F R$ between machines for different $m$-machine $M F R C$ s

\begin{tabular}{|c|c|c|c|}
\hline \multirow[t]{2}{*}{ Travel Time } & \multicolumn{2}{|c|}{ Layout } & \multirow{2}{*}{$\begin{array}{c}\text { Gap } \\
\text { Linear - Circular }\end{array}$} \\
\hline & Linear & Circular & \\
\hline Additive & $|e-f| \delta$ & $\min \{|e-f| \delta,(m+1-|e-f|) \delta\}$ & $\max \{0,(2|e-f|-m-1) \delta\}$ \\
\hline Euclidean & $\begin{array}{c}\delta_{\mathrm{ef}}: \delta_{\mathrm{ef}}<\delta_{\mathrm{eg}}+\delta_{\mathrm{gf}} \\
\forall g \in\{\min \{e, f\}+1, \ldots, \max \{e, f\}-1\}\end{array}$ & $\begin{array}{c}\delta_{\mathrm{ef}}: \delta_{\mathrm{ef}}<\delta_{\mathrm{eg}}+\delta_{\mathrm{gf}} \\
\forall g \in\{1, \ldots, m+1\} \backslash\{e, f\}\end{array}$ & $\begin{array}{c}\max \left\{0, \delta_{\mathrm{ef}}\right\}: \delta_{\mathrm{ef}}<\delta_{\mathrm{eg}}+\delta_{\mathrm{gf}}, \\
\forall g \in\{1, \ldots, \min \{e, f\}-1, \max \{e, f\}+1, \ldots, m+1\}\end{array}$ \\
\hline Constant & $\delta$ & $\delta$ & 0 \\
\hline
\end{tabular}

Table 3. Travel time saving of circular two-machine MFRCs with additive travel time metric

\begin{tabular}{|cccccccc|}
\hline & \multicolumn{3}{c}{ Linear } & & \multicolumn{3}{c|}{ Circular } \\
\cline { 2 - 4 } \cline { 7 - 8 }$S_{k j}^{\sigma(l)}$ & Additive & Euclidean & Constant & & Additive & Euclidean & Constant \\
\hline$S_{11}^{\sigma(l)}$ & $3 \delta$ & {$[\delta, 3 \delta]$} & $\delta$ & & 0 & 0 & 0 \\
$S_{12}^{\sigma(l)}$ & $2 \delta$ & {$[0,2 \delta]$} & 0 & & 0 & 0 & 0 \\
$S_{21}^{\sigma(l)}$ & $3 \delta$ & {$[\delta, 3 \delta]$} & $\delta$ & & 0 & 0 & 0 \\
$S_{22}^{\sigma(l)}$ & $2 \delta$ & {$[0,2 \delta]$} & 0 & & 0 & 0 & 0 \\
\hline
\end{tabular}

The arguments in Table 3 go as follows. For the linear case with additive travel time, rewriting Equations (3)-(6) leads to the corresponding column in the table. This is because we need an extra $3 \delta$ between $A_{2}^{\sigma(l-1)}$ and $A_{0}^{\sigma(l)}$ for each one of $f_{2}\left(S_{11}^{\sigma(l)}, \gamma^{\sigma(l)}\right)$ and $f_{2}\left(S_{21}^{\sigma(l)}, \gamma^{\sigma(l)}\right)$ in Equations (3) and (5). Likewise, for each part $l$ where $l \in$ MPS, we require an extra $\delta$ between $A_{2}^{\sigma(l-1)}$ and $A_{1}^{\sigma(l)}$ and another extra $\delta$ between $A_{1}^{\sigma(l)}$ and $A_{0}^{\sigma(l)}$ for each one of $f_{2}\left(S_{12}^{\sigma(l)}, \gamma^{\sigma(l)}\right)$ and $f_{2}\left(S_{22}^{\sigma(l)}, \gamma^{\sigma(l)}\right)$ in Equations (4) and (6). For the linear case with Euclidean travel time, there is a chance to have a better performance, such that only an extra $\delta$ between $A_{2}^{\sigma(l-1)}$ and $A_{0}^{\sigma(l)}$ is needed for each one of $f_{2}\left(S_{11}^{\sigma(l)}, \gamma^{\sigma(l)}\right)$ and $f_{2}\left(S_{21}^{\sigma(l)}, \gamma^{\sigma(l)}\right)$ in Equations (3) and (5). Under ideal condition, we also require no extra $\delta$ between $A_{2}^{\sigma(l-1)}$ and $A_{1}^{\sigma(l)}$ (and $A_{1}^{\sigma(l)}$ and $A_{0}^{\sigma(l)}$ ) for each one of $f_{2}\left(S_{12}^{\sigma(l)}, \gamma^{\sigma(l)}\right.$ ) and $f_{2}\left(S_{22}^{\sigma(l)}, \gamma^{\sigma(l)}\right)$ in Equations (4) and (6). Note that the ideal condition of the linear case with Euclidean travel time is when it has a constant travel time. In what follows, we consider the cases that the problem is equivalent to circular two-machine MFRCs with additive travel time metric.

Theorem 3. Regardless of the travel time metric, the optimization of the circular case is equivalent of the optimization of circular two-machine $M F R C$ s with multiple parts types and additive travel time metric. 
Proof. Recalling Equations (3)-(6), there is no transit between non-adjacent location pairs. In other words, any pairs chosen from $I / O$, machine $M_{1}$ and machine $M_{2}$ consists a pair of adjacent locations. This is enough to conclude that all elapsed travel times are equal to $\delta$, as it is the case for constant travel time metric. Therefore, we can conclude that Theorems 1 and 2 generally remain valid for a circular case $\square$.

The final note is that the above theorem is crucial since small-scale circular MFRCs are more common in practice and they need less space of shop floor than linear ones. In addition, stationary robotic arms are more economical to setup and easier to program that keep them as a robust solution as comparison with Cartesian MFRs.

\section{Conclusion remarks}

The performance analysis of small-scale robotic systems becomes very complex when stochastic variables such as controllable testing times are taken into consideration, especially for the case of non-identical parts production. Analyzing the performance of such problems under a scalarized formulation has shown that optimal solutions depends on operational parameters $a_{\sigma(l)}, b_{\sigma(l)}, \delta$ and quality parameters $p_{\sigma(l)}$ and $\gamma^{\sigma(l)}$. Thus, we accomplish the highest benefit by assuming the problem as an integrated one to generate optimal cycles according to $a_{\sigma(l)}, b_{\sigma(l)}, \delta, p$ and $\gamma^{\sigma(l)}$. Finally, as a design problem, pickup criterion of the robotic system is studied. Due to a set of feasibility constraints, the search spaces for scheduling problems were reduced for no-wait and interval pickup criteria. Moreover, considering travel time metric, a preliminary analysis has identified the regions where the performance of a circular two-machine system is more than that of the corresponding linear system. The results prove that the circular layout dominates the linear layout and the travel time metric of the system with circular layout is not an important issue. As a research direction, we can consider dual-gripper robots that are very common in practice. For such a robot, the NDT test can be performed by each of two grippers.

\section{References}

[1] Safaei, N. and Tavakkoli-Moghaddam, R. (2009), Integrated multi-period cell formation and subcontracting production planning in dynamic cellular manufacturing systems, International Journal of Production Economics, 120(2) 301- 314.

[2] Tingelstad, L. and Egeland, O. (2014), Robotic assembly of aircraft engine components using a closed-loop alignment process, Procedia CIRP, 23(1) 110-115.

[3] Kumar, S.V., Mani, V.G.S. and Devraj, N. (2014), Production planning and process improvement in an impeller manufacturing using scheduling and OEE techniques, Procedia Materials Science, 5(1)1710-1715.

[4] Brauner, N. (2008), Identical part production in cyclic robotic cells: concepts, overview and open questions, Discrete Applied Mathematics, 156(3) 2480-2492.

[5] Liu, S.Q. and Kozan, E. (2017), A hybrid metaheuristic algorithm to optimise a real-world robotic cell, Computers and Operations Research, 84(1) 188-194.

[6] Elmi, A. and Topaloglu, S. (2018), Cyclic job shop robotic cell scheduling problem: Ant colony optimization, Computers and Industrial Engineering, 111(1) 417-432.

[7] Yan, P., Liu, S.Q., Sun, T. and Ma, K. (2018), A dynamic scheduling approach for optimizing the material handling operations in a robotic cell, Computers and Operations Research, 99(1) 166-177. 
[8] Sethi, S.P., Sriskandarajah, C., Sorger, G., Blazewicz, J. and Kubiak, W. (1992), Sequencing of parts and robot moves in a robotic cell, International Journal of Flexible Manufacturing. Systems, 4(1) 331-358.

[9] Agnetis A. (2000), Scheduling no-wait robotic cells with two and three machines, European Journal of Operational Research, 123 (1) 303-314.

[10] Batur, D.G., Karasan, O.E. and Akturk, M.S. (2012), Multiple part-type scheduling in flexible robotic cells, International Journal of Production Economics, 135(2) 726-740.

[11] Elmi, A., and Topaloglu, S.A. (2013), Scheduling problem in blocking hybrid flow shop robotic cells with multiple robots, Computers and Operations Research, 40(10) 2543-2555.

[12] Batur, D.G., Erol, S. and Karasan, O.E. (2016), Robot move sequence determining and multiple part-type scheduling in hybrid flexible flow shop robotic cells, Computers and Industrial Engineering, 100(1) 72-87.

[13] Gultekin, H., Coban, B. and Akhlaghi, V.E. (2018), Cyclic scheduling of parts and robot moves in m-machine robotic cells, Computers and Industrial Engineering, 90(1) 161-172.

[14] Gultekin, H., Akturk, M.S. and Karasan, O.E. (2008), Bicriteria robotic cell scheduling, Journal of Scheduling, 11(6) 457-473.

[15] Gultekin, H., Akturk, M.S. and Karasan, O.E. (2010), Bicriteria robotic operation allocation in a flexible manufacturing cell, Computers and Operations Research, 37(4) 779-789.

[16] Foumani, M., Gunawan, I., Smith-Miles, K. and Ibrahim, Y. (2015), Notes on feasibility and optimality conditions of small-scale multi-function robotic cell scheduling problems with pick up restrictions, IEEE Transaction on Industrial Informatics, 11(3) 821-829.

[17] Foumani, M., Gunawan, I. and Smith-Miles, K. (2015), Increasing throughput for a class of two-machine robotic cells served by a multifunction robot, IEEE Transactions on Automation Science and Engineering, 14(2) 1150-1159.

[18] Foumani, M., Razeghi, I. and Smith-Miles, K. (2020), Stochastic optimization of twomachine flow shop robotic cells with controllable inspection times: From theory toward practice, Robotics and Computer Integrated Manufacturing, in press.

[19] Romauch, M. and Hartl, R.F. (2017), Capacity planning for cluster tools in the semiconductor industry, International Journal of Production Economics, 194 (1) 167-180.

[20] Madathil, S.C. Nambiar, S. Mason, S.J. and Kurz M.E. (2018), On scheduling a photolithography area containing cluster tools, Computers and Industrial Engineering, 121 (1) $177-188$.

[21] Dawande, M. Geismar N.H. Sethi, S.P. and Sriskandarajah C. (2005), Sequencing and scheduling in robotic cells: recent developments, Journal of Scheduling, 8 (5) 387-426.

[22] Fazel Zarandi, M.H. Mosadegh, H. and Fattahi M. (2013), Two-machine robotic cell scheduling problem with sequence dependent setup times, Computers and Operations Research, 40 (5) 1420-1434.

[23] Geismar, H.N. and Pinedo, M. (2010), Robotic cells with stochastic processing times, IIE Transactions, 42(12) 897-914.

[24] Logendran, R. and Sriskandarajah, C. (1996), Sequencing of robot activities and parts in twomachine robotic cells, International Journal of Production Research, 34(12), 3447-3463.

[25] Giri, B.C. and Dohi, T. (2007), Inspection scheduling for imperfect production processes under free repair warranty contract, European Journal of Operational Research, 183(1), 238252.

[26] Ross, S.M. (1996) Stochastic Processes, 2nd ed. New York: Wiley (1996).

[27] Applegate, D.L., Bixby, R.E., Chvatal, V. and Cook, W.J. (2006), The traveling salesman problem: a computational study, Princeton University Press. 12(5) 1-10.

[28] Kabadi, S. N. and Fazle Baki, Md. (1999), Gilmore-Gomory type traveling salesman problems, Computers and Operations Research, 26(1) 329-351. 
[29] Foumani, M. Smith-Miles, K. Gunawan, I. and Moeini A. (2017), A framework for stochastic scheduling of two-machine robotic rework cells with in-process inspection system, Computers and Industrial Engineering, 112(1) 492-502.

[30] Jolai, F., Foumani, M., Tavakoli-Moghadam R. and Fattahi, P. (2012), Cyclic scheduling of a robotic flexible cell with load lock and swap, Journal of Intelligent Manufacturing, 23(5) 1885-1891. 